\title{
REVIEW
}

\section{Hemodynamic changes in infective endocarditis}

\author{
BRUNO BARŠıĆ
}

$\operatorname{BRUNO} \operatorname{BARSIC}(\bowtie)$

Intensive Care Unit

Hospital for Infectious Diseases School of Medicine

Mirogojska 8, 10000 Zagreb

Phone: + 38514603404

Fax: + 38514603222

E-mail: bruno.barsic@fran.bfm.hr

\begin{abstract}
Hemodynamic disturbances in patients with infective endocarditis (IE) are complex and might be consequences of various pathogenic processes. Congestive heart failure (CHF) is a major contributor of hemodynamic derangements, but since IE is often a septic disease, reversible myocardial dysfunction as well as septic shock might sometimes dominate or represent additional contributing factors of disturbed hemodynamics. The major cause of CHF is malfunction of affected valves. It occurs with an incidence of $32 \%$ and is often severe. This is why CHF is the most important risk factor associated with poor patient outcome. Results of the project 'International Collaboration on Endocarditis' related to the characteristics of CHF in patients with IE are presented.
\end{abstract}

Key words: infective endocarditis, congestive heart failure, sepsis, Staphylococcus aureus

Infective endocarditis (IE) is still a serious disease with a high mortality (16 $-20 \%$ ) overall. It is a localized infection involving the heart valves in the majority of patients, but it is also often a generalized infection associated with the systemic inflammatory response syndrome and numerous septic metastases in various parts of the body. Due to this, there is a diversity of pathogenic mechanisms involved in the hemodynamic changes in IE.

The clinical and microbiological spectra of IE have been evolving continually from the first descriptions of the disease at the beginning of the 20th century. The changing profile of IE is nowadays well established and documented (table 1). (1-3)

During the first seventy years of the twentieth century IE was usually of a subacute clinical course because less pathogenic bacteria, like alpha-hemoIytic streptococci of the viridans group, dominated as major pathogens. They did not induce a pronounced inflammatory response. Rheumatic valve dis- ease was the major underlying cause. Nowadays, the course of IE is mostly acute, septic and the most common pathogen is Staphylococcus aureus. The incidence of enterococcal or Streptococcus bovis infections has risen. Atherosclerotic valvular disease is the most common form of valvular disease due to the increased incidence of degenerative valvular lesions associated with an ageing population. IE occurs in patients without previously known cardiac disease in up to almost $50 \%$ of patients. The average age of patients with IE has increased over time resulting in a shift towards the eighth or even ninth decade of life. Other more prevalent risk factors associated with $\mathrm{IE}$, are patients with implanted intracardiac devices (pacemakers, defibril- lators) although, fortunately, there is a decreased incidence in patients with prosthetic valve infective endocarditis (PVIE). A horrific increase in the number of intravenous drug addicts has led to a greater incidence of right-sided IE.

Recently, some other epidemiological characteristics of IE have emerged. Although community-acquired infections still dominate, health care associated infections are growing, particularly those associated with dialysis, implantation of Hickman's and Broviac catheters and other invasive procedures. (4) All these changes affect the hemodynamics in patients with IE in regard to its pathogenesis and incidence (figure 1). While hemodynamic derangements associated with valvular or paravalvular dysfunctions are well known, changes

Table 1. Changing aspects of IE.

- Increasing average age

- Lower number of patients with previously recognized valve disease

- Decrease in the incidence of PVIE

- Increase in the incidence of S. Aureus and BHS 'D' infections

- Health care associated IE increased

- IE in iv. addicts increased

- Earlier cardiac surgery

- Better prognosis 


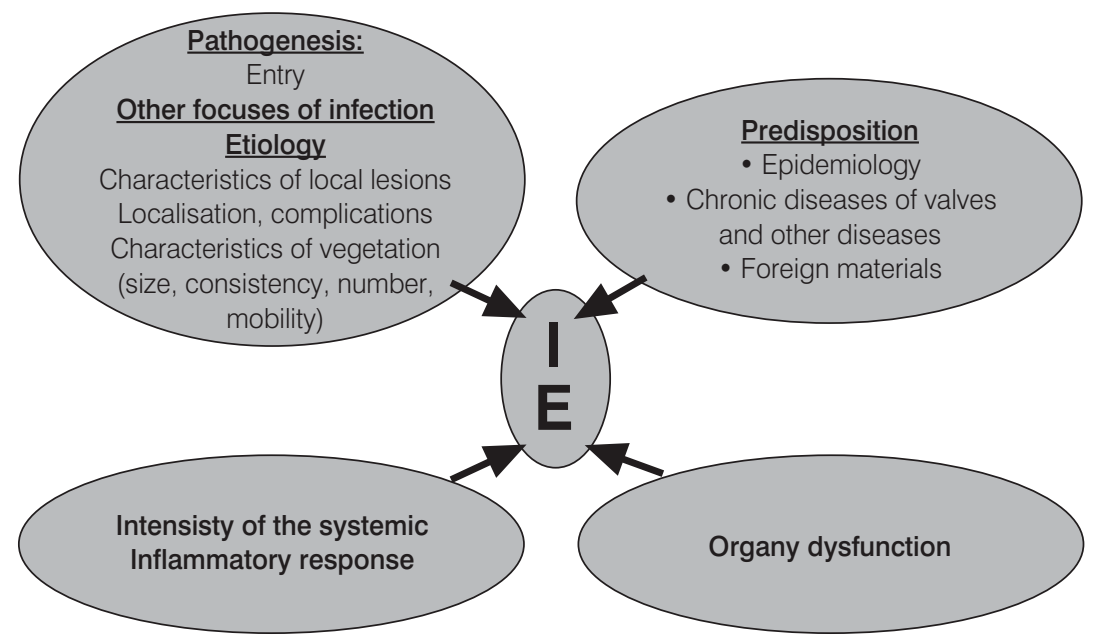

Figure 1. Pathogenesis of hemodynamic derangements in patients with infective endocarditis (IE).

associated with systemic inflammatory response syndrome (SIRS) and organ dysfunctions are not adequately addressed in clinical research of IE. It is often forgotten that IE is only one segment of a generalized, acute systemic inflammatory response - sepsis.

The most established hemodynamic changes are associated with infections of valves and surrounding tissues and consequent valve dysfunction. The most important and most severe complication is congestive heart failure (CHF) which may rapidly progress due to the dysfunction of heart valves (rapid progression of aortic and/or mitral regurgitation), valve perforation, formation of a paravalvular abscess and fistulation, paravalvular leak due to dehiscence of prosthetic valves (table 2). Congestive heart failure demands earlier surgical intervention, which is one of the most important factors today contributing to a better prognosis.

$\mathrm{CHF}$, along with embolizations, is the most common complication of IE. The incidence of $\mathrm{CHF}$ in patients with IE is $30 \%$ to $40 \%$. Despite this, CHF has not been particularly evaluated in separate studies because it is a well-known complication with few unresolved questions. Our center in the Hospital for Infectious Diseases is actively involved in a project known as the 'International Collaboration on Endocarditis Prospec-
Does CHF occur more often in elderly patients? Despite a slight tendency towards a greater incidence in the group of patients older than 74 years, the difference was not statistically significant (figure 3). This is in accordance with other ICE reports.

One might expect that because of a worse prognosis for patients with PVIE, the incidence of $\mathrm{CHF}$ is higher compared to native valve disease. We were not able to confirm this. The incidence of CHF was 621 out of 1906 (32.6\%) patients with NVIE and 175 out of 564 (31\%) patients with PVIE.

Is localization of IE associated with CHF? Sexton and al. showed that aortic valve disease is more commonly associated with $\mathrm{CHF}$ than mitral valve involvement (28\% vs. 20\%). (5) In our group of patients with left-sided NVIE the incidence of CHF was significantly higher if both valves were affected (53.7\%) than if only the aortic valve $(37.5 \%)$ or mitral valve $(31.1 \%)$ were involved.

Since $\mathrm{S}$. aureus is the most common pathogen causing IE today with a worse prognosis than IE caused by other bacteria, we asked if S. aureus IE was associated with a greater incidence of CHF. Interestingly, there was a significant increase in the incidence of $\mathrm{CHF}$ among patients with staphylococcal NVIE (34.1\% vs. $29.4 \%, p=0.04)$. That was not observed in PVIE (figure 4). There was no difference in the incidence of CHF in patients with IE caused with MSSA compared to MRSA.

Patients with CHF were more commonly subjected to cardiac surgery than patients without CHF (62\% vs. 38.9\%, $p<0.001$ in NVIE and $55.1 \%$ vs. $45.4 \%$,

Table 2. Hemodynamic derangements in patients with IE.

\begin{tabular}{ll}
\hline Valve dysfunction & Changes associated with sepsis \\
\hline - Regurgitation & • Hyperdynamic phase \\
- Perforation & - Reversible myocardial dysfunction \\
- Rupture of chordae mitralis & - Septic shock \\
- Stenosis & \\
- Intracardiac shunt & \\
- Paravalvular 'leak' (dehiscence) & \\
- Fistulation (abscess) & \\
\hline
\end{tabular}




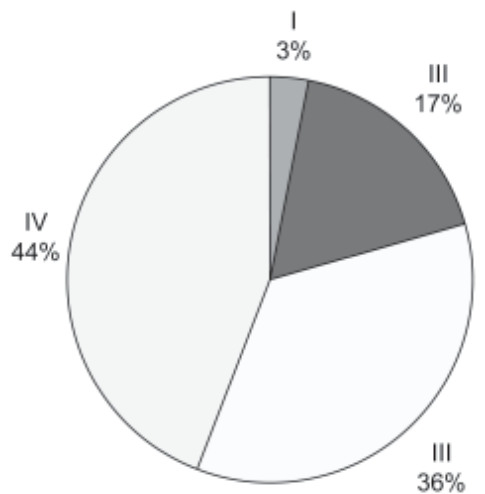

Figure 2. Severity of congestive heart failure (CHF) according to NYHA classification in patients with infective endocarditis (ICE study).

$\mathrm{p}<0.05$ in patients with PVIE). Cardiac surgery, in a number of studies, has been associated with better patient survival and $\mathrm{CHF}$ is often the commonest indication for surgery. Intra-hospital mortality was significant- ly higher amongst patients with $\mathrm{CHF}$ (27.5\% vs. $11.1 \%$ in NVIE and $33.5 \%$ vs. $18.3 \%$ in PVIE). Other ICE studies confirmed that $\mathrm{CHF}$ is the most important independent factor associated with intra-hospital death in patients with IE. These data explain why hemodynamic complications are, along with centralnervous system embolizations, the most important factor that necessitates admission of patients with IE to intensive care units (ICU). The incidence of $\mathrm{CHF}$ in patients admitted to ICU is $28 \%$ to $64 \%$. $(6,7)$

Because of the striking evidence of the association between $\mathrm{CHF}$ and poor patient survival, other aspects of hemodynamic derangement have been less evaluated. It is often forgotten that IE is in the majority of patients a septic disease. Changes in peripheral vascular resistance, cardiac output and blood pressure add to derangements associated with valve failure. Revers-

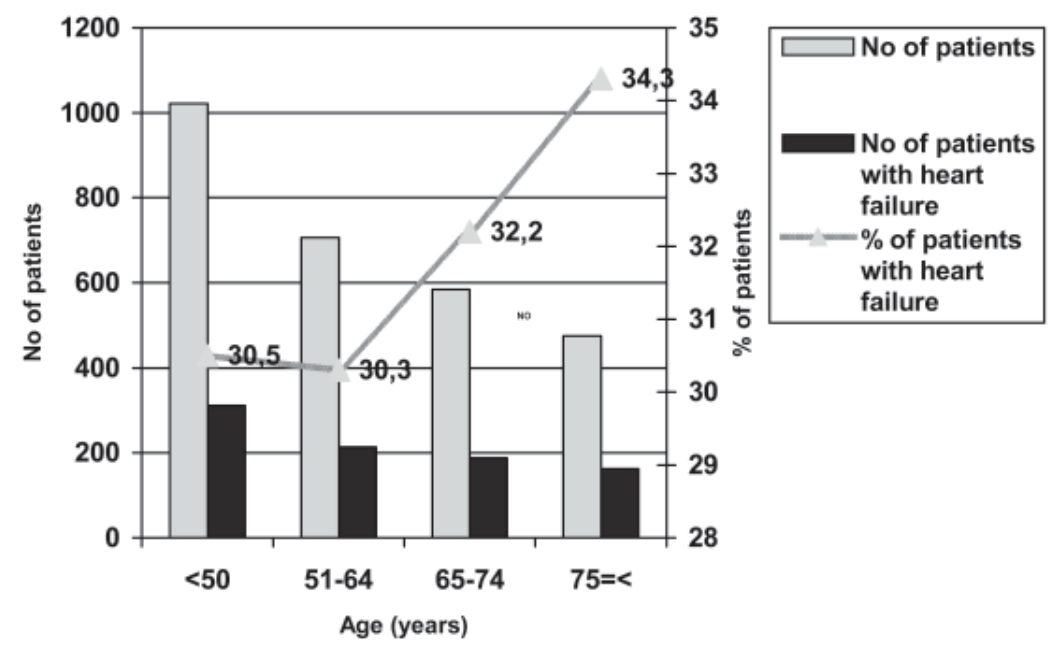

Figure 3. Influence of age on the incidence of congestive heart failure in patients with infective endocarditis (ICE study). ible myocardial dysfunction is further imposed on existing disturbances and should be taken into consideration in patients with IE. Severe CHF is associated with pulmonary edema, which is further complicated by ARDS and capillary leak syndrome, which accompany

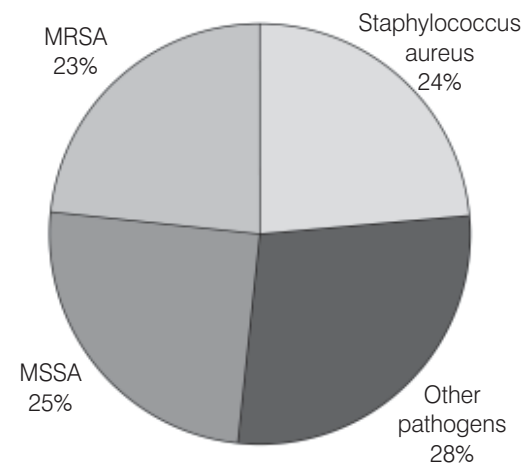

Figure 4. Congestive heart failure in patients with staphylococcal endocarditis (ICE study).

severe sepsis and septic shock. Central nervous system complications might be further complicated by neurogenic shock and renal failure with volume overload. All these hemodynamic consequences of IE are often superimposed on chronic cardiac diseases, particularly in elderly patients.

The complexity of hemodynamic changes in patients with IE is also reflected in therapeutic options in septic patients. It is clear how treatment with fluids and sympatomymetics must be well balanced as well as invasive organ support such as mechanical ventilation and applied renal replacement therapy.

However, many problems still remain and many aspects of IE need further clarification.

\section{REFERENCES}

1. Hoen B, Alla F, Selton-Suty C, Béguinot I, Bouvet A, Briançon S, et al, editors. Association pour l'Etude et la Prévention de l'Endocardite Infectieuse (AEPEI) Study Group. Changing profile of infective endocarditis: results of a 1-year survey in France. JAMA 2002;288:75-81.

2. Delahaye F, Goulet V, Lacassin F, Ecochard R, Selton-Suty C, Hoen B, et al, editors. Characteristics of infective endocarditis in France in 1991. A 1-year survey. Eur Heart J 1995;16:394-401. 
3. Fefer P, Raveh D, Rudensky B, Schlesinger Y, Yinnon AM. Changing epidemiology of infective endocarditis: a retrospective survey of 108 cases, 1990-1999. Eur J Clin Microbiol Infect Dis 2002;21:432-7.

4. Fowler VG Jr, Miro JM, Hoen B, Cabell CH, Abrutyn E, Rubinstein E, et al, editors. Staphylococcus aureus endocarditis: a consequence of medical progress. JAMA 2005;293:3012-21.

5. Sexton DJ, Spelman D. Current best practices and guidelines: assessment and management of complications in infective endocarditis. Cardiol Clin 2003;21:273-282.

6. Karth G, Koreny M, Binder T, Knapp S, Zauner C, Valentin A, Honninger R, et al, editors. Complicated infective endocarditis necessitating ICU admission: clinical course and prognosis. Crit Care 2002;6:149-154.

7. Mourvillier B, Trouillet JL, Timsit JF, Baudot J, Chastre J, Régnier B, et al, editors. Infective endocarditis in the intensive care unit: clinical spectrum and prognostic factors in 228 consecutive patients. Intensive Care Med 2004;30:2046-52. 